This item was submitted to Loughborough's Research Repository by the author.

Items in Figshare are protected by copyright, with all rights reserved, unless otherwise indicated.

\title{
Airport noise simulation using neural networks
}

PLEASE CITE THE PUBLISHED VERSION

PUBLISHER

(C) IEEE

VERSION

VoR (Version of Record)

LICENCE

CC BY-NC-ND 4.0

\section{REPOSITORY RECORD}

Yang, Yingjie, Chris J. Hinde, and David Gillingwater. 2019. "Airport Noise Simulation Using Neural Networks". figshare. https://hdl.handle.net/2134/4123. 
This item was submitted to Loughborough's Institutional Repository (https://dspace.lboro.ac.uk/) by the author and is made available under the following Creative Commons Licence conditions.

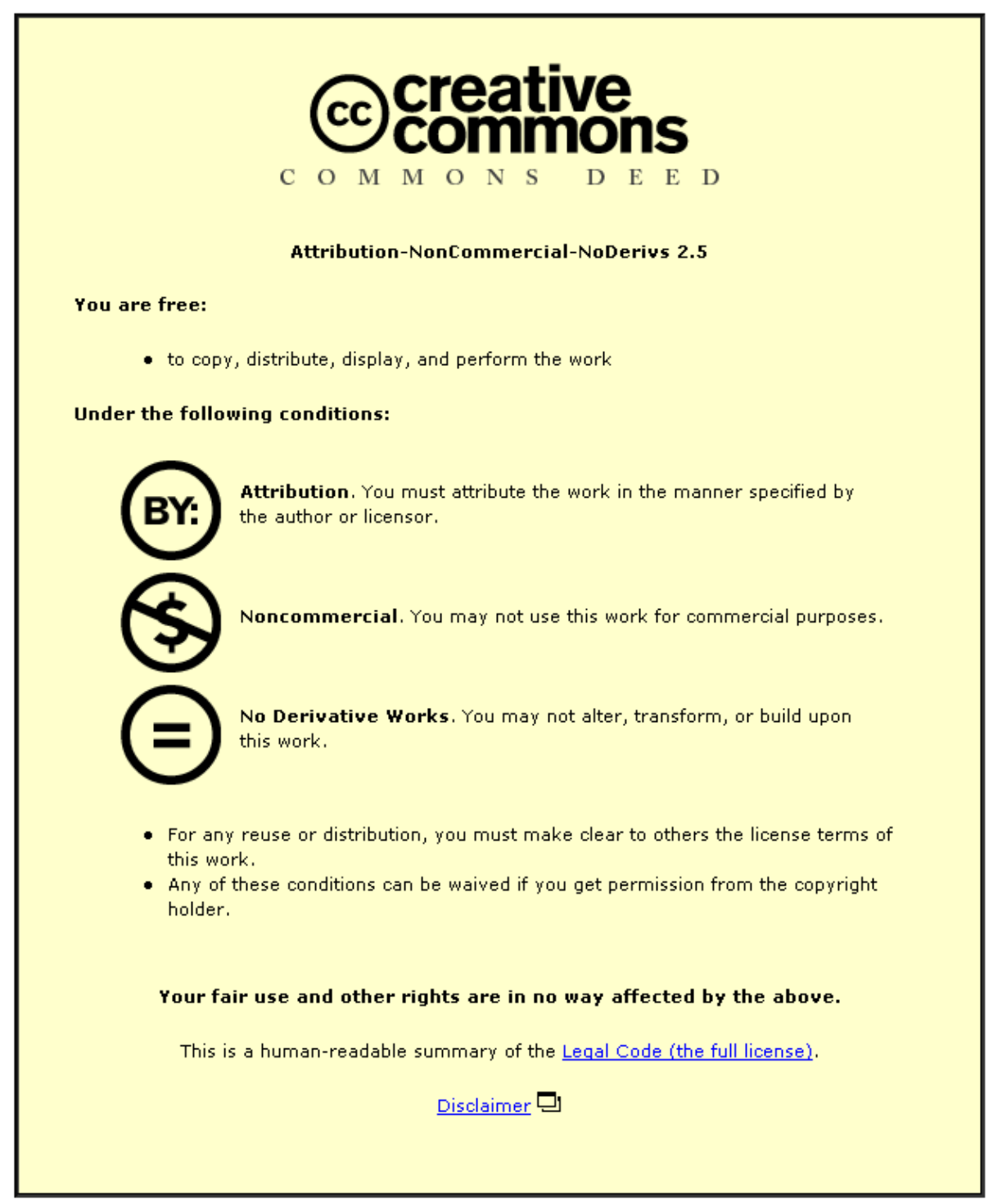

For the full text of this licence, please go to: http://creativecommons.org/licenses/by-nc-nd/2.5/ 


\title{
Airport Noise Simulation Using Neural Networks
}

\author{
Yingjie Yang*, Chris Hinde** and David Gillingwater**
}

\begin{abstract}
Aircraft noise is influenced by many complex factors and it is difficult to devise an accurate mathematical model to simulate it with respect to operations at an airport. This paper presents an investigation in simulating airport noise using artificial neural networks. The results show that it is possible to establish a simple neural network model with monitored data for a specific airport and specific aircraft under local conditions.
\end{abstract}

\section{INTRODUCTION}

In airport operations, identifying and monitoring noise disturbance caused by a specific aircraft movement at a specific airport on a specific local community is very important when considering financial penalties, compensation claims and social costs [1], [2]. Aircraft noise around an airport is influenced by many factors, such as its geographical location and the location of aircraft in 3-dimensional space, the power thrust of an aircraft's engines at any given moment, the number of engines on an aircraft, its weight and speed, as well as wind speed and direction, ambient air temperature and geographical features around the point at which aircraft noise is being measured. This list is not complete, and there are many more factors that affect real airports. These differ also from airport to airport, hence the relationships are very complex.

The standard methodologies available follow one of two classes: (i) the 'laboratory model' - based on laboratory-type experiments and standardized in-situ tests undertaken in given conditions; or (ii) the 'replication/simulation model' - based primarily on in-situ test data. However, in practice it is not feasible to monitor each impacted locality around an airport and the interactions between the key factors are too complicated to enable reliable mathematical models to be developed. As a result, laboratory models are in fact the dominant models in use. For instance, aircraft noise calculations around airports are dominated by calibrated parametric models based on standard condition tests and aircraft engine manufacturers' data, such as the US FAA integrated noise model, the INM [3] . These standard models are very useful in simulation analysis at a general level, but they suffer from difficulties in incorporating specific

*Y. Yang is with the Centre for Computational Intelligence, Faculty of Computing Sciences and Engineering, De Montfort University, The Gateway, Leicester, LE1 9BH, UK yyangedmu.ac.uk

**C. Hinde is with the Department of Computer Sciences, Loughborough University, Loughborough, LE11 3TU, UK C.J.Hinde@lboro.ac.uk

***D. Gillingwater is with the Transport Studies Group, Department of Civil and Building Engineering, Loughborough University, Loughborough, LE11 3TU, UK D.Gillingwaterelboro.ac.uk local conditions; thus a location-specific model established with data from that site is likely to yield more realistic results although its generalizability is likely to be poor when compared with the laboratory model.

A neural network excels at learning from data and does not require the prior specification of a mathematical model. This feature makes it an ideal candidate in environmental analysis where a large amount of monitoring data exists but where the interactive mechanisms are too complex or little understood to specify an accurate mathematical model. At those airports where awareness of the significance of environmental impacts like aircraft noise is increasing, more and more environmental data are monitored but collected only from a very limited number of geographical locations. These data can provide a basis for the application of neural networks. However, there is no real world evidence so far in the precision of neural networks in simulating noise levels at airports, hence it is necessary to have real world case studies to verify it. This paper investigates the applicability of neural networks in simulating noise levels at airports, based on a set of data from Manchester airport in the UK.

\section{STRUCTURE OF NEURAL NETWORKS AND SIGNIFICANCE ANALYSIS}

One of the difficulties in establishing a neural network (NN) is the determination of its structure. It is a common understanding that only the simplest network structure can give the best solution. Therefore, various network pruning technologies have been developed [4]-[6]. However, one of the key features in neural networks is that they perform complicated analyses or mapping by means of a combination of huge amounts of simple neurons [7]. The real biological world does not necessarily rely on strict mathematics or pruning technology to run their activities, but they do display such an array of perfect functions that scientific method may never be able to explain adequately. The 'compound eye' of an insect [8] is just one of these amazing facts: in addition to its ability to accommodate overlapping inputs, it involves many other different mechanisms which makes it impossible to simulate with only a simple structure. This fact does not exclude the notion that simple overlapped inputs may contribute to its powerful functionality.

Based on this idea, a simple approach to making use of overlapped or redundant inputs to improve the training results of NN was put forward in [9], see Figure 1. This method employs multiple input nodes for the same input 
parameter in the network structure and simulates their influences in the compound eye of insects [10] by a random initialisation of their connecting weights. Although this network is equivalent to a conventional network convergence is faster and more accurate [9].

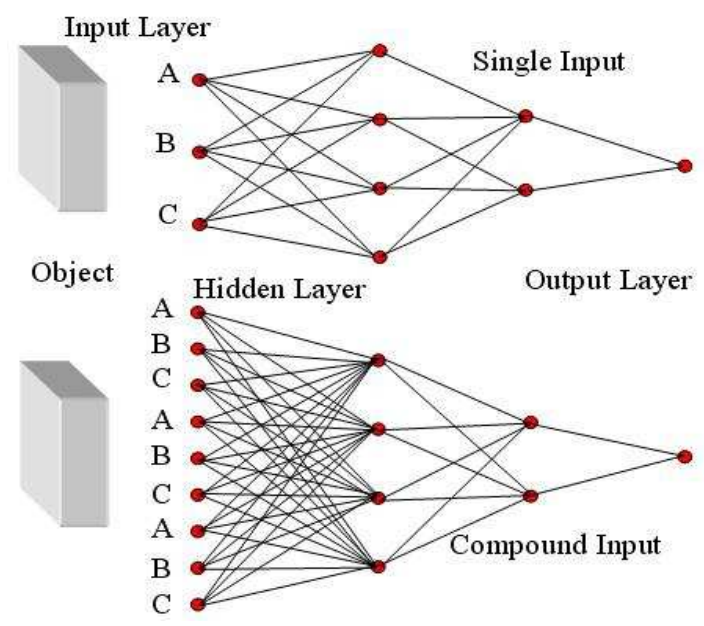

Fig. 1. Redundant input NN and ordinary NN

Having established a neural network, it is necessary to evaluate its performance. In addition to the usual methods of verifying with data not in the training sets, we put forward an approaches based on GRSE and GPRSE in [11].

Definition 1 (Global Relative Strength of Effect (GRSE)): For a given sample set $S=\left\{s_{1}, s_{2}, s_{3}, \ldots, s_{j}, \ldots, s_{r}\right\}$, where, $s_{j}=\{X, Y\}, X=\left\{x_{1}, x_{2}, x_{3}, \ldots, x_{p}\right\}$, $Y=\left\{y_{1}, y_{2}, y_{3}, \ldots, y_{q}\right\}$, if there is a neural network trained by BP algorithm with this set of samples, the $R S E_{k i}$ exists as

$$
\begin{aligned}
G R S E_{k i}= & C \sum_{j_{n}} \sum_{j_{n-1}} \ldots \sum_{j_{1}} W_{j_{n} k} W_{j_{n-1} j_{n}} \\
& W_{j_{n-2} j_{n-1}} W_{j_{n-3} j_{n-2}} \ldots W_{i j_{1}}
\end{aligned}
$$

where $C$ is a normalized constant which regulates the maximum absolute value of $G R S E_{k i}$ as 1 .

Definition 2 (Global Potential RSE (GPRSE)): : For a neural network trained using the BP algorithm and for a given reference data set $S=\left\{s_{1}, s_{2}, s_{3}, \ldots, s_{j}, \ldots, s_{r}\right\}$, where, $s_{j}=\{X, Y\}, X=\left\{x_{1}, x_{2}, x_{3}, \ldots, x_{p}\right\}, Y=$ $\left\{y_{1}, y_{2}, y_{3}, \ldots, y_{q}\right\}$ :

$$
\operatorname{GPRSE}_{k i}=\frac{\sum_{j_{n}} \sum_{j_{n-1}} \cdots \sum_{j_{1}}\left|W_{j_{n} k}\right|\left|W_{j_{n-1} j_{n}}\right| \cdots\left|W_{i j_{1}}\right|}{\sum_{i} \sum_{j_{n}} \sum_{j_{n-1}} \cdots \sum_{j_{1}}\left|W_{j_{n} k}\right|\left|W_{j_{n-1} j_{n}}\right| \cdots\left|W_{i j_{1}}\right|}
$$

where, $\mathrm{W}$ is a connected weight and $\mathrm{e}$ is the input value in its corresponding node.

According to the value of $G R S E_{k i}$, we can assess how much influence the input unit has on the output unit. The more the weight is revised due to the input unit, the larger the variance of the weights becomes linked to this input unit. Because the original values of the weights are similar, the larger are the absolute values of the weights, the more the effect of the input unit will have on the output. So, the $G R S E_{k i}$ shows the global dominance of input on output.

GPRSE is a measure of the absolute value of every weight and node value. The absolute influence of every connection and node is thus accumulated. Hence, no matter which factors are dominant, the contribution of every factor will be incorporated within the calculation of GPRSE. Compared with GRSE, the removal of the different signs makes the GPRSE less sensitive to a small change of input, thus it is a measure of the potential within a wider scope of neighbourhood rather than a detailed trend at a specific point.

\section{NOISE EVALUATION USING NEURAL NETWORKS}

Noise disturbance at airports is currently the most significant environmental problem, and most airports are adopting noise models for their operational planning. As aforementioned, most existing noise models are based on some standard tuned data sets. For example, INM adopts what are called NPD data sets as its foundation [12]. Normal NPD data consist of two or more noise curves [3]. A noise curve reflects the relationship between distance (D) and noise levels $(\mathrm{N})$ under specific engine power $(\mathrm{P})$ (thrust in pounds) and operational mode (departure or approach) under standard conditions. However, these curves give only measurements at the following distances: 200, 400, 630, 1000, 2000, 4000, 10000, 16000 and 25000 feet. Any noise level between these measurements or between those given thrusts has to be evaluated using mathematical models, such as linear interpolation, logarithmic interpolation and extrapolation. However, these mathematical models are established against a standard measuring environment at a specific site for the test. The geographical conditions and environmental parameters at other airports may not be the same as at the testing site, so models established in INM may not give results as near to the real world measurement as expected. To adjust those parameters in INM to suit the local geographic and environmental condition at an airport is complicated and difficult, and there are many mathematical models involved into these processes to consider the relationship between noise level and temperature, wind speed and direction, and other acoustics factors. Due to the complexity of the natural environment at an airport, these models cannot fit every airport and are bound to produce further errors and uncertainty. Therefore, a simple way of establishing noise simulation at a local airport would be a great help in airport noise simulation and operational planning. Here, we adopt neural networks as the universal models for adapting standard NPD curves to local conditions. 


\section{A. Available data}

Because of the significant impact of aircraft noise on airport development, most large airports in the world already monitor the noise levels in their vicinity. With the incorporation of Manchester airport in our EPSRC-funded research into a decision support system for sustainable airport development, we collected a large set of monitored noise records.

These data were monitored during the period 1998-2001, and the largest volume were recorded for one aircraft type, the Boeing B757: 10408 records from Kell House Farm. The recorded data attributes include aircraft type, operation mode, direct distance to the monitoring station, station name, maximum noise level recorded and its recording time. Considering the volume of the data, we show it by its noise distribution against distance under departure or approach operation in Figure 2 and 3.

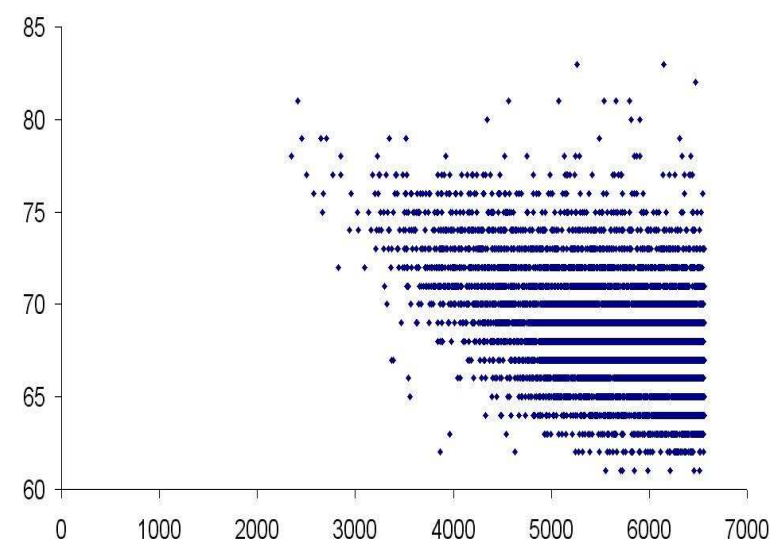

Fig. 2. Noise distribution against distance for departing flights recorded at Kell House Farm station

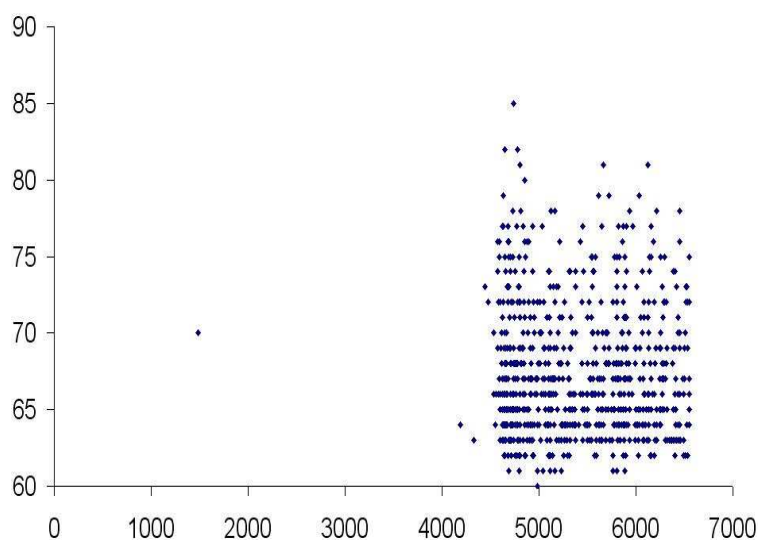

Fig. 3. Noise distribution against distance for approaching flights recorded at Kell House Farm station

The monitored data in Figure 2 and 3 are scattered around the same distances. Obviously, a general model suitable to each airport is very difficult to establish for such data set without knowing more information about the flight speed, thrust, weight, trajectory, wind speed and direction, terrain of the airport etc. Among these factors, the weather conditions and geographical features of the vicinity of an airport would not be as different as those between two airports. The monitored data are measured under local weather and geographical conditions, hence their influence on the noise levels around the airport has already been embedded into the measurements. A model established from the monitored data is suitable only for the airport where data are collected. Therefore, weather and geographical conditions are not as significant as distance and thrust for a local noise model, in which case, NPD curves are accurate as long as the weather and geographical conditions at a local airport match those conditions of the standard test site. A critical disagreement between INM model results and in-situ monitoring data comes from the difference between their treatment of weather and geographical conditions. Therefore, a local NPD curve could be established considering the same relationships as INM: the relationships between noise levels, operation modes, distances and thrust. Other factors are not significant for the observations in the vicinity of the same airport. The data in Figure 2 and 3 have attributes for noise levels, distances and operation mode. However, thrust is missing in the collected data set. In actual fact, for the same distance at the airport and observation location, there is more than one point in both figures. This is mainly caused by their different thrust measurements at that distance. Because of the automated landing control deployed by an aircraft on final approach, the thrust is often changed during the approach operation and causes larger fluctuation of the points in Figure 3 than 2. It demonstrates that thrust is a significant factor determining the monitored noise levels. Therefore, it is essential to obtain thrust data for those monitored points in Figure 2 and 3. However, thrust is not recorded in the monitored data, and it is very difficult to obtain it due to the large number of possible flight trajectories.

\section{B. Reverse map thrust using neural networks and NPD data}

We made an attempt to establish neural networks without thrust data, but the result was not satisfactory. The result was especially poor when a network trained using data from one monitoring station was applied to the other station. They were not better than an average estimation in most cases. It proves again that thrust data is essential in the noise evaluation at airports. Now that NPD curves provide the relationships among noise levels, operation modes, distances and thrust, it is possible to establish a model to create a reverse map to find thrust from known noise levels as well. Neural networks provide ideal tools in doing this reverse mapping from the available standard NPD curves. There will be differences between the actual thrust and those measured in flights. However, it is possible to 
establish mathematical models to adjust an actual thrust into a measured thrust. Compared with the adjustment of noise for every concerned location involving a huge variety of factors, it is much easier to adjust a single thrust from a single aircraft engine.

Based on the aforementioned idea, we need to establish the reverse map from known noise levels to their corresponding thrusts. Before establishing the reverse map, we need to evaluate the capacity of neural networks in mapping the NPD curves. We adopt the data from the standard NPD database in INM here. The NPD databases in INM contain a set of NPD data for 224 aircraft types. There are four kinds of NPD noise data:

- $L_{A E}$ A-weighted sound exposure level;

- $L_{A S m x}$ Maximum A-weighted sound level with slowscale exponential time weighting;

- $L_{E P N}$ Effective tone-corrected perceived noise level;

- $L_{P N T S m x}$ Maximum tone-corrected perceived noise level with slow-scale exponential time weighting.

Here, to match our monitored data, we adopt only the maximum A-weighted sound level with slow-scale exponential time weighting $L_{A S m x}$. The aim of this experiment is to simulate NPD curves using neural networks so as to obtain those missing thrust values in our data set. For this experiment, we require the same aircraft type as the one with the maximum number of available monitored data records. Here, the aircraft determined by our monitored data is the Boeing B757. Therefore, we adopt NPD data for this aircraft only.

NPD data for each aircraft are very limited and we have to make full use of available data. Here, the "leave-out-one cross validation" method is adopted in the training of neural networks. We adopt a compound input of 10 sets and set the hidden layer node number as 6 . The inputs are operational mode (OP_MODE), maximum noise level (Noise_Level) and distance; the output is thrust. After 30000 iterations using "leave-out-one cross validation", the errors are reduced to less than 2.0E-4. The cross validation results show that the maximum difference is less than $14 \%$, and over $88 \%$ of rows have differences less than $5 \%$. Table I gives the number of records with errors lower than the given error in the first column (\%) and their corresponding accuracy. The result is also demonstrated in Figure 4.

These results demonstrate that the trained neural network is a valid method to derive thrust from measured noise levels. From the trained neural network, we can obtain the GRSE and GPRSE as shown in Table II.

Distance plays the dominant role in determining thrust, and noise has an important role too. Aircraft operations do not seem to be very significant for thrust. These conclusions agree with our data set: a longer distance and lower noise level indicates less thrust from aircraft engines. The
TABLE I

MAPPING RESULTS OF THE REVERSE NEURAL NETWORK FROM NPD DATA

\begin{tabular}{|c|c|c|}
\hline $\begin{array}{l}\text { Error } \\
(\%)\end{array}$ & $\begin{array}{l}\text { Lower error } \\
\text { records }\end{array}$ & Accuracy (\%) \\
\hline 0 & 60 & 0.00 \\
\hline 1 & 46 & 23.33 \\
\hline 2 & 32 & 46.67 \\
\hline 3 & 20 & 66.67 \\
\hline 4 & 11 & 81.67 \\
\hline 5 & 6 & 90.00 \\
\hline 6 & 5 & 91.67 \\
\hline 7 & 4 & 93.33 \\
\hline 8 & 4 & 93.33 \\
\hline 9 & 3 & 95.00 \\
\hline 10 & 3 & 95.00 \\
\hline 15 & 0 & 100.00 \\
\hline
\end{tabular}

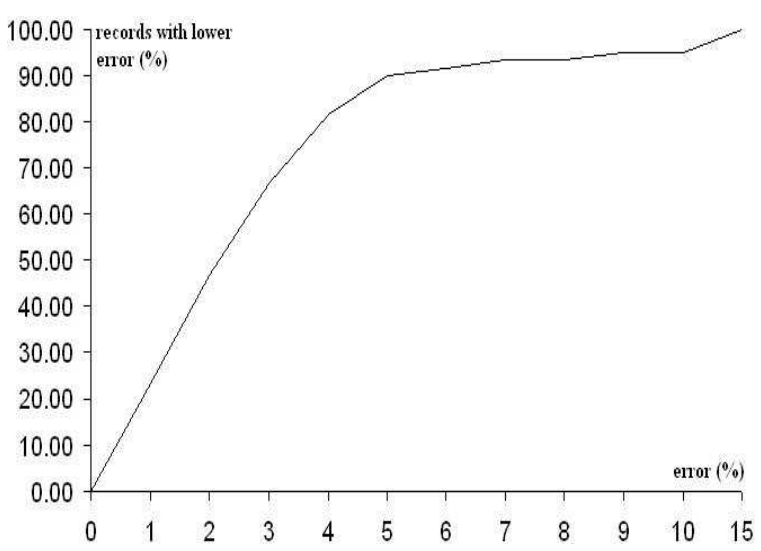

Fig. 4. Mapping results of the revers mapping neural network from NPD data

operational mode determines if thrust is stable or not, but it does not determine its values. According to our model in evaluating trained neural networks [11], the network obtained here is acceptable.

Using the trained neural networks, we obtained the missing thrusts for the monitored data. As suggested by GRSE, distance is the dominant factor of the thrust values, so we demonstrate their distribution against distance in Figure 5 and 6.

Data in Figure 5 and 6 demonstrate very high correspondence to data in Figure 2 and 3. The noise level values in Figure 2 and 3 show some linear patterns, and these result in the curve patterns in Figure 5 and 6. For the same noise level, thrust values increase with distance. For the same distance, thrust increases with noise values and jumps from one curve to the curve above it. Obviously, it is the same as that has been revealed by GRSE and GPRSE. It thus demonstrates the efficiency of GRSE and GPRSE. 
TABLE II

GRSE AND GPRSE FOR A NEURAL NETWORK ESTABLISHED FROM NPD DATA OF B757

\begin{tabular}{|l|l|l|}
\hline \hline Factor & GRSE & GPRSE \\
\hline Operation mode & -0.02 & 0.03 \\
Noise level & 0.23 & 0.20 \\
Distance & 1.0 & 0.78 \\
\hline
\end{tabular}

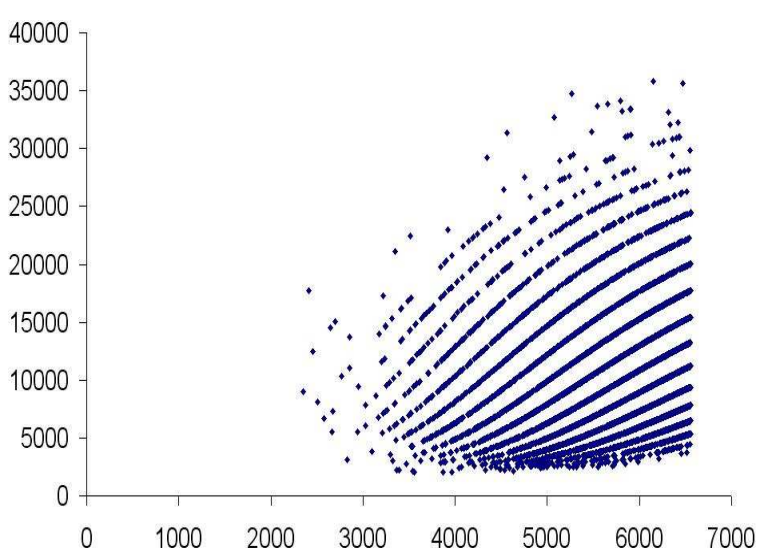

Fig. 5. Thrust distribution against distance for departing flights over Kell House Farm station

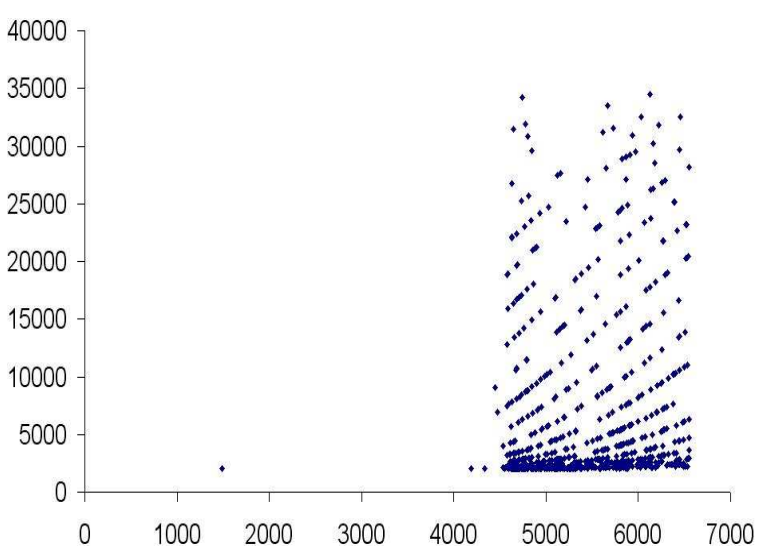

Fig. 6. Thrust distribution against distance for approaching flights over Kell House Farm station

\section{Noise level prediction using neural networks}

Having obtained the thrust data for each record, we establish a neural network using the measured data at Kell House Farm station. Similar to the NPD network, we adopt the same input parameters: distance between an aircraft and the monitoring station, operational mode of the aircraft and its thrust. The output is the maximum noise level at the monitoring station. For the sake of speed, we use 10 sets of inputs again in the compound structure. We again use 10 sets of inputs as compound inputs and 8 hidden layer nodes.
TABLE III

Noise LeVel testing Result for Kell House Farm station

\begin{tabular}{|c|c|c|}
\hline $\begin{array}{l}\text { Difference } \\
(\mathrm{dBA})\end{array}$ & $\begin{array}{l}\text { Records with } \\
\text { higher difference }\end{array}$ & $\begin{array}{l}\text { Records with } \\
\text { lower difference } \\
(\%)\end{array}$ \\
\hline 0 & 4208 & 0 \\
\hline 0.05 & 3251 & 37.53 \\
\hline 0.1 & 1879 & 63.89 \\
\hline 0.5 & 160 & 96.92 \\
\hline 1 & 65 & 98.75 \\
\hline 1.5 & 18 & 99.65 \\
\hline 2 & 9 & 99.82 \\
\hline 3 & 3 & 99.94 \\
\hline 5 & 1 & 99.98 \\
\hline
\end{tabular}

There are 10408 records for the B757 at Kell House Farm station. We separate the data into two different groups: each record with an odd index number is held as training data, and each row with an even index number is kept as testing data. In this way, we have 5204 rows in both groups. After 5000 iterations, the error is reduced to less than $1.0 \mathrm{e}-4$. The testing results are shown in Table III and Figure 7.

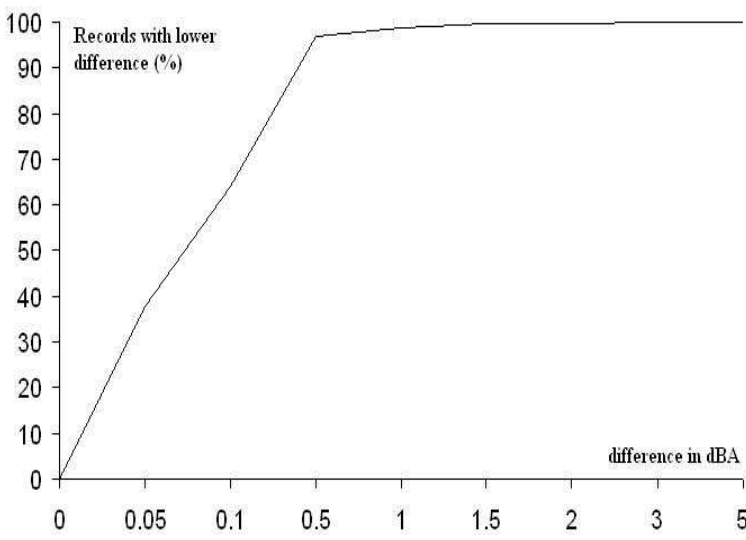

Fig. 7. Noise prediction against data from Kell House Farm station

In Table III and Figure 7, the "difference" refers to the difference between the measured noise level and the output noise level from the trained neural network. The "records with lower difference (\%)" represent the percentage of records with a noise level lower than the corresponding difference. It is obvious that the percentage of records with a lower difference is very high for differences over 0.5 dBA. It is therefore a very accurate representation of noise levels at an airport. With the use of thrust, it is possible to obtain a very high accuracy in the prediction of noise levels at an airport.

It would be interesting to know if the NPD model is sufficiently good in doing this. To test the capacity of a 
TABLE IV

NOISE LEVEL TESTING RESUlt FOR KELl HOUSE FARM STATION USING NPD NETWORK

\begin{tabular}{lll}
\hline \hline $\begin{array}{l}\text { Difference } \\
(\mathrm{dBA})\end{array}$ & $\begin{array}{l}\text { Records } \\
\text { higher difference }\end{array}$ & $\begin{array}{l}\text { with } \\
\text { lower } \\
(\%)\end{array}$ \\
\hline 0 & 5204 & 0 \\
0.05 & 5008 & 3.77 \\
0.1 & 4794 & 7.88 \\
0.5 & 3129 & 39.87 \\
1 & 1379 & 73.50 \\
1.5 & 638 & 87.74 \\
2 & 301 & 94.22 \\
3 & 93 & 98.21 \\
5 & 16 & 99.69 \\
\hline \hline
\end{tabular}

neural network trained from NPD data, we established a similar model using NPD data. The structure of the network is exactly the same as the network for Kell House Farm station. Using the "leave one out" cross validation method, we established the NPD neural network for noise level. Applying this NPD neural network, we obtained test results for the test data at Kell House Farm station as shown in Table IV and Figure 8.

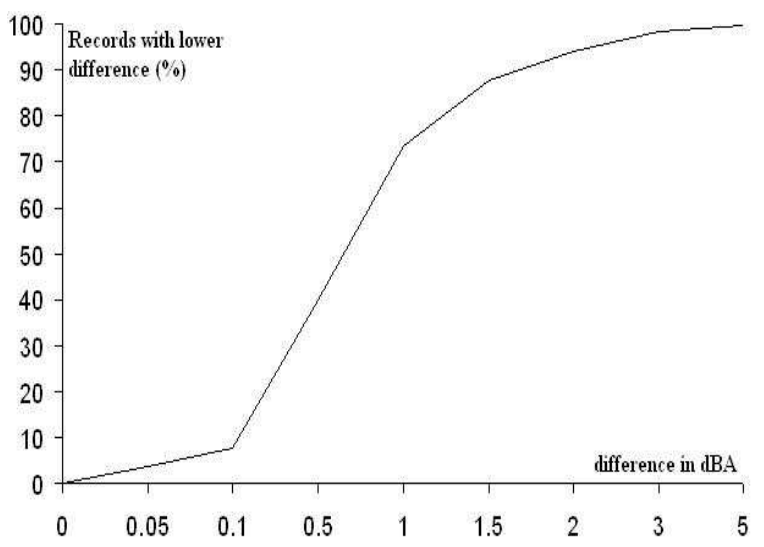

Fig. 8. Noise prediction against data from Kell House Farm station using NPD network

Comparing Figure 7 and 8, it is clear that the neural network trained with NPD data could only provide a reasonable prediction $(70 \%)$ when the difference is $1 \mathrm{dBA}$ or above, and its prediction is very poor for $0.5 \mathrm{dBA}(40 \%)$. However, the neural network trained with data measured at Kell House station could provide much better results for both data sets.

The poor results from the model trained with NPD data are caused by differences between the geographical and weather conditions of the standard measuring environment at the NPD test site and the conditions pertaining at
TABLE V

GRSE AND GPRSE OF THE TRAINED NEURAL NETWORKS USING NPD DATA AND Kell House FARM DATA

\begin{tabular}{|l|l|l|l|l|}
\hline \hline \multirow{2}{*}{ Input } & \multicolumn{2}{|c|}{ NPD } & \multicolumn{2}{c|}{ Kell House Farm } \\
\cline { 2 - 5 } & GRSE & GPRSE & GRSE & GPRSE \\
\hline Operation mode & -0.014 & 0.049 & 0.012 & 0.085 \\
Distance & -1 & 0.772 & -0.064 & 0.1 \\
Thrust & 0.171 & 0.179 & 1 & 0.815 \\
\hline \hline
\end{tabular}

Manchester airport. This complex relationship is very difficult to tune with INM, but relatively straightforward using neural networks. The data are measured at Manchester airport, so its values have already reflected the geographical and weather conditions there. Although the trained model is not applicable to other airports, it achieves a better performance at the local airport.

The GRSE and GPRSE values of the two models trained with NPD data and Kell House Farm data are shown in Table V.

The data in Table $\mathrm{V}$ show that the dominant factor in the NPD model is distance, but that this changes to thrust in the model trained with Kell House Farm data. Whereas NPD data are obtained under conditions at a test site where the aircraft is being flown to meet aircraft cerification standards, the monitored data come from various operations of aircraft when they take off or land at an airport under different and more complex weather conditions. Therefore, the frequency of the change of their thrust is much higher than under standard testing conditions. This means that the actual noise level is more frequently influenced by thrust rather than distance in real operational conditions. Therefore, the model using NPD data is bound to give larger errors when compared to real operations. It therefore provides evidence that airports could reduce noise levels by improved aircraft operation under similar geographical conditions.

\section{CONCLUSIONS}

Based on our experiments of noise prediction at airports using neural networks trained with in-situ data, it is clear that neural networks can provide a convenient tool in adjusting the standard NPD curves to local conditions and hence a network trained with in-situ data can provide better predictions than models based on standard NPD curves alone. Limited by data availability, we derived thrust data through a reverse mapping neural network simulating standard NPD curves. Such a methodology can be used to modify a standard NPD curve to reflect better the relationships between thrust and noise levels, and it can increase the accuracy of NPD predictions in reality. Similar relationships exist between derived thrust and in-situ noise data. The relative difference between the standard NPD prediction and our neural network prediction is at least the same as shown in this research. 
However, the superiority of neural networks over the standard NPD model at a local airport is obvious.

\section{REFERENCES}

[1] P. Morrell and C. H. Lu, "Aircraft noise social cost and charge mechanisms - a case study of amsterdam airport schiphol," Transportation Research, Part D: Transport and Environment, vol. 5, pp. 305-320, 2000.

[2] J. Nelson, "Meta-analysis of airport noise and hedonic property values," Journal of Transport Economics and Policy, vol. 38, p. 127, 2004.

[3] F. A. Administration, INM 6.0 Technical Manual. Washington, DC: FAA, 2002.

[4] M. Hagiwara, "Removal of hidden units and weights for back propagation networks," in Proceedings of IJCNN, vol. 1, Nagoya, Japan, 1993, pp. 351-354.

[5] R. Reed, "Pruning algorithms - a survey," IEEE Transactions on Neural Networks, vol. 4, no. 5, pp. 740-747, 1991.

[6] R. Setiono, "A penalty function approach for pruning feed-forward neural networks," Neural Computation, vol. 9, pp. 185-204, 1997.

[7] R. Hecht-Nielsen, Neurocomputing. Reading, MA: Addison-Welsley, 1990.

[8] J. M. Karpilow, A. C. Pimentel, H. K. Shamloula, and T. R. Venkatesh, "Neuronal development in the drosophila compound eye: Photoreceptor cells $\mathrm{r} 1, \mathrm{r} 6$, and $\mathrm{r} 7$ fail to differentiate in the retina aberrant in pattern (rap) mutant," Journal of Neurobiology, vol. 31, no. 2, pp. 149-165, 1996.

[9] Y. Yang, C. Hinde, and D. Gillingwater, "Improve neural network training using redundant structure," in Proceedings of the International Joint Conference on Neural Networks 2003 (IJCNN03), 2003, pp. 2023-2027.

[10] I. F. Yang, J. T. Lin, and C. Y. Wu, "Fine structure of the compound eye of mallada basalis (neuroptera: Chrysopidae)," Annals of the Entomological Society of America, vol. 91, no. 1, pp. 113-121, 1998.

[11] Y. Yang, C. Hinde, and D. Gillingwater, "A new method to evaluate a trained artificial neural network," in Proceedings of the International Joint Conference on Neural Networks 2001 (IJCNNO1), Summer 2001, pp. 2620-2625.

[12] T. Connor, "Integrated noise model - the federal aviation administration's computer program for predicting noise exposure around an airport," in Proceedings of the "INTER-NOISE 80", vol. 1, Miami, FL, (USA), 1980, pp. 127-130. 